\title{
Cuidados imediatos aos recém-nascidos pré-termos em um hospital de ensino
}

\author{
Immediate care for premature infants in a teaching hospital \\ Atención inmediata a los recién nacidos prematuros en un hospital universitário
}

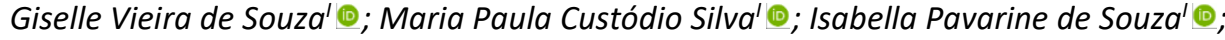

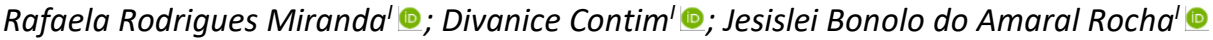

\begin{abstract}
'Universidade Federal do Triângulo Mineiro, Uberaba, Minas Gerais, Brasil
RESUMO

Objetivo: descrever os cuidados imediatos realizados em recém-nascidos pré-termos na sala de parto. Método: estudo observacional, retrospectivo de abordagem quantitativa, realizado em um hospital de ensino. Os dados foram coletados de 144 prontuários de recém-nascidos pré-termos, no período de janeiro de 2019 a janeiro de 2020, e submetidos à análise descritiva e ao teste Qui-quadrado. A pesquisa recebeu aprovação do Comitê de Ética em Pesquisa. Resultados: sobre os cuidados imediatos aos recém-nascidos pré-termo, 52,1\% tiveram clampeamento do cordão umbilical antes do primeiro minuto, 22,2\% realizaram contato pele a pele precoce, $34,7 \%$ iniciaram aleitamento materno precoce, $66,4 \%$ realizaram aspiração de vias aéreas na sala de parto. Conclusão: evidenciou-se que o clampeamento precoce do cordão umbilical, a aspiração de vias aéreas e a utilização de oxigênio inalatório para estabilização clínica são cuidados frequentes realizados nos recém-nascidos pré-termo na sala de parto.
\end{abstract}

Descritores: Neonatologia; Enfermagem Neonatal; Recém-Nascido Prematuro; Salas de Parto.

\section{ABSTRACT}

Objective: to describe the immediate care provided to preterm newborns in the delivery room. Method: this quantitative, retrospective, observational study was conducted at a teaching hospital. Data collected from 144 medical records of preterm newborns, between January 2019 and January 2020, were submitted to descriptive analysis and the Chi-square test. The study was approved by the research ethics committee. Results: immediate care for preterm newborns included the following: $52.1 \%$ had their umbilical cord clamped before the first minute, $22.2 \%$ had early skin-to-skin contact, $34.7 \%$ started breastfeeding within the first hour, and $66.4 \%$ underwent airway aspiration in the delivery room. Conclusion: early umbilical cord clamping, airway aspiration and inhaled oxygen for clinical stabilization were found to be provided in the delivery room frequently in preterm newborns.

Descriptors: Neonatology; Neonatal Nursing; Infant, Premature; Delivery Rooms.

\section{RESUMEN}

Objetivo: describir la atención inmediata realizada a los recién nacidos prematuros en la sala de partos. Método: estudio observacional, retrospectivo, de enfoque cuantitativo, realizado en un hospital universitario. Se colectaron datos de 144 historias clínicas de recién nacidos prematuros, de enero de 2019 a enero de 2020, y se sometieron a análisis descriptivo y prueba de Chi-cuadrado. El estudio fue aprobado por el Comité de Ética en Investigación. Resultados: en cuanto a la atención inmediata a los recién nacidos prematuros, el $52,1 \%$ tuvo pinzamiento del cordón umbilical antes del primer minuto, el 22,2\% tuvo contacto precoz piel a piel, el 34,7\% inició la lactancia materna precoz, el 66,4\% realizó aspiración de la vía aérea en sala de parto. Conclusión: se demostró que el pinzamiento precoz del cordón umbilical, aspiración de las vías respiratorias y el uso de oxígeno inhalado para la estabilización clínica son cuidados frecuentes en los recién nacidos prematuros en la sala de partos. Descriptores: Neonatología; Enfermería Neonatal; Recien Nacido Prematuro; Salas de parto.

\section{INTRODUÇÃO}

A adaptação do recém-nascido (RN) à vida extrauterina requer cuidados específicos de acordo com a idade gestacional e o peso. Assim, os recém-nascidos pré-termos (RNPT) ${ }^{1}$ apresentam maior risco de morbidade e mortalidade, visto que, após o nascimento, são mais susceptíveis à hipotermia, hipoglicemia, hipotensão e insuficiência respiratória, com a consequente adoção de condutas mais intervencionistas².

Anualmente, mais de três milhões RN vão à óbito antes do primeiro mês de vida, dos quais um terço não sobrevive ao primeiro dia de vida ${ }^{3}$. Em países desenvolvidos, como nos Estados Unidos, mais da metade das mortes neonatais ocorrem em RNPT com menos de 32 semanas $^{4}$. No Brasil, a prematuridade é considerada uma causa potencialmente evitável, ocupando o nono lugar entre os países com maior número de RNPT, apresentando um índice de 11,2\% de nascimentos prematuros por ano $0^{5}$. Ademais, entre as principais causas de mortalidade em menores de um ano de idade, destacam-se as complicações neonatais no parto prematuro, antes das 37 semanas, com 25\% no ano de 2016, o que demonstra a necessidade de ações mais efetivas ${ }^{6}$. 
Considerando que a assistência na primeira hora de vida do RPNT impacta diretamente sobre complicações posteriores e tempo de internação em unidades neonatais, deve-se intervir minimamente e priorizar os cuidados essenciais $^{2}$. Neste sentido, recomenda-se o contato pele a pele, a manutenção da temperatura, a permeabilidade de vias aéreas, a estimulação do aleitamento materno (AM) precoce e o clampeamento oportuno do cordão umbilical ${ }^{1}$.

Dada a complexidade e a necessidade de reanimação neonatal, principalmente em menores de 28 semanas, observa-se o adiamento ou a não realização de algumas práticas, como o contato pele a pele, o AM precoce e o clampeamento oportuno do cordão. Entretanto, estes cuidados são capazes de reduzir a mortalidade nesse período e devem ser realizados precocemente sempre que possível².

O contato pele a pele precoce na sala de parto, além de ser um ato instintivo da mã ${ }^{1}$, proporciona o aumento da temperatura materna contribuindo para a manutenção da temperatura do RN entre 36,5ํ C e 37으. Além disso, ressaltase que a proteção e promoção do calor diminuem o consumo de glicose e oxigênio dos RNPT, evitando intercorrências como a hipoglicemia, disfunção respiratória e hipotermia ${ }^{7,8}$.

Com a finalidade de manter a permeabilidade das vias aéreas nos RNPT propõem-se o uso de coxim sob os ombros, a fim de manter o pescoço em leve extensão diante da identificação de excesso de secreções em vias aéreas e da necessidade de aspiração, respeitando-se a pressão máxima aproximada de $100 \mathrm{mmHg}$, com uso de sonda traqueal seguindo a ordem da boca e depois as narinas, delicadamente ${ }^{9}$.

O AM precoce proporciona a ingestão do colostro, que é altamente nutritivo, de fácil digestão e rico em benefícios imunológicos. Dentre os inúmeros benefícios desta prática, aponta-se o fortalecimento do vínculo, a melhor adaptação à vida extrauterina e o favorecimento da regulação glicêmica, cardiorrespiratória e térmica ${ }^{10}$. Logo após o parto, o RN apresenta um período de reatividade, com comportamento inato de sugar o seio materno, que estimula a amamentação na primeira hora de vida ${ }^{11}$. Esta sucção precoce, ao aumentar a produção de ocitocina e prolactina, contribui para o estímulo à produção e ejeção do leite materno, além de reduzir o risco materno de sangramento pós-parto ${ }^{10}$.

O clampeamento oportuno do cordão umbilical, entre 30 e 60 segundos após o nascimento, é recomendado para todos os RN, inclusive os RNPT vigorosos. Porém, nos casos de reanimação na sala de parto, não existe consenso ${ }^{12}$, ainda que esta prática se associe com a redução de hipoglicemia neonatal, hemorragia intraventricular, retinopatia da prematuridade e displasia broncopulmonar ${ }^{13}$.

A despeito da importância dos cuidados imediatos aos RN na sala de parto e das recomendações para a assistência neste contexto assistencial, observa-se que muitos profissionais de saúde não priorizam estes cuidados, sobretudo em relação aos RNPT, levando-os precocemente para avaliação no local de reanimação ${ }^{8}$.

Diante desta problemática e compreendendo que a adoção de protocolos baseados nas melhores evidências é fundamental para nortear os profissionais de saúde na implementação de boas práticas na assistência neonatal, o presente artigo tem como objetivo descrever os cuidados imediatos realizados em recém-nascidos pré-termos na sala de parto.

A relevância deste estudo reside na possibilidade de fornecer subsídios para a avaliação da assistência neonatal e o planejamento de capacitações profissionais, com vistas ao alinhamento dos cuidados imediatos às recomendações baseadas em evidências, sobretudo em relação aos RNPT.

\section{MÉTODO}

Estudo observacional retrospectivo, com abordagem quantitativa, realizado em um hospital de ensino do estado de Minas Gerais, com os prontuários dos RNPT nascidos vivos, norteado pelas recomendações do Strengthening the Reporting of Observational Studies in Epidemiology (STROBE) da rede EQUATOR.

Os critérios de inclusão adotados foram: ser recém-nascido pré-termo e ter nascido vivo no referido hospital, no período de janeiro de 2019 a janeiro de 2020. Ressalta-se que os prontuários não localizados ou identificados foram excluídos.

A coleta de dados ocorreu no período de dezembro de 2020 a janeiro de 2021, iniciando com o levantamento dos prontuários junto ao Setor de Gestão de Processos e Tecnologia da Informação da instituição. Os dados de interesse para a pesquisa foram as variáveis relacionadas à caracterização materna e obstétrica (procedência, idade materna e tipo de parto), bem como variáveis relacionadas ao RN (sexo, índice de APGAR, peso ao nascer, idade gestacional, gemelaridade, presença de malformações congênitas e classificação quanto à idade gestacional). Também se considerou as variáveis relacionadas aos cuidados imediatos: clampeamento do cordão umbilical, contato pele a pele, amamentação, complicações mais frequentes (desconforto respiratório/apneia/gasping, frequência cardíaca < 100bpm, líquido amniótico meconial, hipotonia) e procedimentos para estabilização do RNPT (intubação orotraqueal, pressão 
positiva contínua das vias aéreas (CPAP) e oxigênio inalatório/hood). Em relação à hospitalização, utilizou-se as seguintes variáveis: local de encaminhamento do RNPT, tempo e desfecho da hospitalização.

Os dados dos prontuários foram inseridos em uma planilha eletrônica do programa Excel $^{\circledR}$ para Windows ${ }^{\circledR}$, por dupla entrada para processamento e análise. Em seguida, os dados foram exportados para o programa Statistical Package for the Social Science (SPSS), versão 23 para Windows ${ }^{\circledR}$, e realizada a análise descritiva das variáveis quantitativas usando medidas como média, desvio-padrão e valores mínimo e máximo. As variáveis categóricas foram descritas a partir de suas distribuições de frequência absoluta e percentual. Realizou-se análise bivariada e teste de Quiquadrado de Pearson, considerando valor de $p<0,005$.

O estudo cumpriu as exigências referentes aos aspectos éticos, obedecendo as diretrizes e normas regulamentadoras de pesquisa envolvendo seres humanos. Foi aprovado por Comitê de Ética em Pesquisa e solicitado a dispensa do Termo de Consentimento Livre e Esclarecido, por se tratar de estudo retrospectivo e ser inviável a sua aquisição.

\section{RESULTADOS}

Foram localizados e incluídos 144 prontuários de RNPT, que atendiam aos critérios de inclusão. Em relação às características maternas e obstétricas, constatou-se que $52,8 \%(n=76)$ das mulheres eram procedentes da Macrorregião de Uberaba, com média da idade materna de 27 anos ( $D P=7,32$ ), com o mínimo de 15 e máximo de 44 anos, sendo que $50,7 \%$ ( $n=73$ ) foram submetidas à cesariana.

No tocante às variáveis relacionadas ao RNPT, identificou-se que: $50 \%(n=77$ ) eram do sexo feminino; $79,8 \%$ ( $n=111$ ) apresentaram boa vitalidade no primeiro minuto de vida e $92,9 \%(n=131)$ no quinto minuto de vida; com média de peso ao nascer de $2295 \mathrm{~g}(\mathrm{DP}=658$ ), sendo no mínimo $495 \mathrm{~g}$ e no máximo $4120 \mathrm{~g} ; 91,6 \%$ ( $\mathrm{n}=131)$ eram adequados para idade gestacional (AIG), 4,9\% $(n=7)$ eram pequenos para idade gestacional (PIG), 3,5\% ( $n=5)$ eram grandes para idade gestacional (GIG); 45,1\% ( $n=65)$ tinham idade gestacional entre 36 semanas e 36 semanas e 6 dias; $16,7 \%(n=24)$ eram gemelares; e 5,6\% $(n=8)$ apresentaram malformações congênitas. De acordo com a classificação da idade estacional, $4,2 \%(n=6)$ dos RNPT eram pré-termos extremos, $10,4 \%(n=15)$ muito pré-termos, $6,9 \%(n=10)$ prétermos moderados e $78,5 \%(n=113)$ pré-termos tardios.

Dos cuidados realizados durante a primeira hora de vida, verificou-se que o tempo de clampeamento do cordão umbilical foi menor que um minuto em $52,1 \%(n=75)$ dos RNPT; $1,4 \%(n=2)$ foram secados e aquecidos junto à mãe; $22,2 \%(n=32)$ realizaram contato pele a pele precoce contínuo; e o AM precoce aconteceu em $34,7 \%(n=50)$.

O desconforto respiratório foi a complicação mais frequente, presente em 47,9\% $(n=69)$ dos RNPT, seguido por hipotonia 9,2\% ( $n=42)$ e frequência cardíaca menor que $100 \mathrm{bpm} 9 \%(n=13)$. A Tabela 1 descreve os procedimentos realizados para a estabilização dos RNPT.

TABELA 1: Distribuição dos procedimentos realizados nos RNPT na primeira hora de vida ( $n=144)$. Uberaba, MG, Brasil, 2019.

\begin{tabular}{lcc}
\hline Variáveis & $\mathbf{n}$ & $\mathbf{\%}$ \\
\hline Aspiração das vias áreas & 93 & 64,6 \\
Aspiração gástrica & 31 & 21,5 \\
O2 inalatório & 37 & 25,7 \\
IOT & 18 & 12,5 \\
CPAP & 4 & 2,8 \\
\hline Fonte: Elaborada pela autora, 2021. \\
Legendas: IOT: Intubação Orotraqueal.
\end{tabular}

A aspiração das vias aéreas foi identificada em $64,6 \%(n=93)$ e a necessidade de terapia inalatória com auxílio de oxigênio em $25,7 \%(n=37)$.

No que se refere à hospitalização do RNPT, após os cuidados e procedimentos na sala de parto, $67,9 \%(n=95)$ dos RNPT foram encaminhados para o Alojamento Conjunto $(A C), 15 \%(n=21)$ para a Unidade de Cuidados Intermediários (UCIN), 12,1\% ( $n=17$ ) para a Unidade de Terapia Intensiva Neonatal (UTIN) e $5 \%(n=7)$ para o Pronto Socorro Infantil (PSI). O tempo médio de hospitalização foi de 15 dias (DP = 26,79), com mínimo de zero e máximo de 184 dias. Quanto ao desfecho, $93,7 \%(n=134)$ tiveram alta e 9,3\% $(n=9)$ foram à óbito.

A Tabela 2 apresenta os resultados das análises estatísticas inferenciais. 
TABELA 2: Análise da variável tempo de clampeamento do cordão umbilical e classificação de idade gestacional, de acordo com os procedimentos dos RNPT na sala de parto $(n=144)$. Uberaba, MG, Brasil, 2021.

\begin{tabular}{lcccc}
\hline & \multicolumn{3}{c}{ Intubação Orotraqueal } \\
Variáveis & Sim & $\mathbf{N a ̃ o}$ & Total & *p valor \\
& $\mathbf{n}(\mathbf{\%})$ & $\mathbf{n}(\mathbf{\%})$ & $\mathbf{n}(\mathbf{\%})$ & $\mathbf{( < 0 , 0 0 5 )}$ \\
\hline Tempo de clampeamento do cordão umbilical & & & & \\
Menor que um minuto & $17(22,7)$ & $58(77,3)$ & $75(100)$ & $p<0,001$ \\
Entre um e três minutos & $1(1,7)$ & $59(98,3)$ & $60(100)$ & $p<0,001$ \\
Maior que três minutos & 0 & $9(100)$ & $9(100)$ & $p<0,001$ \\
Classificação de idade gestacional & & & & \\
Pré-termo extremo - menor que 28 semanas & $6(100)$ & 0 & $6(100)$ & $p<0,001$ \\
Muito pré-termo - 28 a 31 semanas & $10(66,7)$ & $5(33,3)$ & $15(100)$ & $p<0,001$ \\
Pré-termo moderado - 32 a 33 semanas & $1(10)$ & $9(90)$ & $10(100)$ & $p<0,001$ \\
Pré-termo tardio - 34 a 36 semanas e 6 dias & $1(0,9)$ & $112(99,1)$ & $113(100)$ & $p<0,001$ \\
\hline Total & $\mathbf{1 8 ( 1 2 , 5 )}$ & $\mathbf{1 2 6 ( 8 7 , 5 )}$ & $\mathbf{1 4 4 ( 1 0 0 )}$ & \\
\hline Fonte: Elaborada pelos autores, 2021. & & & &
\end{tabular}

Após investigação de associação entre os dados relacionados ao tempo de clampeamento do cordão umbilical e os procedimentos realizados na sala de parto, mediante análise bivariada e realização de teste de Qui-quadrado, considerando valor de $p<0,005$, identificou-se que dos RNPT que foram submetidos a intubação orotraqueal (IOT), $22,7 \%(n=17)$ tiveram o cordão umbilical com clampeamento menor que um minuto e $66,7 \%$ ( $n=10$ ) eram RNPT muito pré-termo.

\section{DISCUSSÃO}

O cuidado com a saúde do RN tem importância fundamental para a redução da mortalidade infantil, a promoção da qualidade de vida e a recuperação da saúde e do bem-estar, sendo uma das prioridades na assistência à saúde infantil, garantindo o crescimento e desenvolvimento adequados nos aspectos físico, emocional e social ${ }^{14,15}$.

Os cuidados imediatos devem ser priorizados com intuito de implementar a promoção da qualidade de vida destes RNPT $^{2}$. Neste estudo, observou-se que $52,1 \%(n=75)$ dos RNPT foram submetidos ao clampeamento precoce do cordão umbilical, o que poderia estar relacionado aos procedimentos necessários à estabilização clínica. Contudo, somente $12,5 \%$ ( $n=144)$ necessitaram de IOT, valor condizente com um estudo correlato sobre reanimação em sala de parto, onde $12,2 \%$ dos RNPT realizaram o mesmo procedimento ${ }^{16}$.

Em RNPT, a prática do clampeamento oportuno é preconizada de 30 a 60 segundos após o nascimento, sendo um cuidado imediato que se associa com a redução da mortalidade, da ocorrência de hemorragia intraventricular e transfusões de hemácias, visto que o adiamento da secção do cordão favorece a estabilidade da pressão arterial e contribui para uma transição cardiorrespiratória mais tranquila ${ }^{17-19}$. No entanto, diante da necessidade de reanimação na sala de parto, ainda não há um consenso ${ }^{12}$.

Em relação à secagem do $\mathrm{RN}$, recomenda-se que esta prática aconteça sobre o tórax materno, seguida da colocação de uma touca dupla de algodão, a fim de evitar a perda de calor por evaporação durante a realização dos demais cuidados imediatos, tais mais como o contato pele a pele e o AM precoce na primeira meia hora de vida9 .

Por outro lado, nos casos de dificuldades no início da respiração ou tônus ineficiente, orienta-se o uso de fonte de calor radiante concomitante às manobras de reanimação, com a utilização de colchão térmico ou saco de poliuretano, sem secagem prévia do $\mathrm{RN}^{9}$. Fato esse observado onde somente $1,4 \%$ dos RNPT foram secados no colo da mãe, o que pode se justificar pelas complicações clínicas e que contraindicariam este cuidado.

Ainda que o contato pele a pele, precoce e ininterrupto, após o parto seja preconizado pela Organização Mundial de Saúde (OMS), os resultados deste estudo mostram a baixa prevalência deste cuidado entre RNPT (22,2\%), assim como identificado em outra investigação, que constatou 17,5\%. Esses dados sugerem a existência de barreiras para realização do contato pele a pele precoce, as quais podem estar relacionadas às condições desfavoráveis maternas e/ou neonatais, bem como à necessidade de transferência ${ }^{20,21}$.

Na prática, observa-se que o contato pele a pele não é priorizado como um cuidado imediato ao RNPT, mesmo quando o mesmo não necessita de transferência imediata para a UTI, não apresentou infecção ou desconforto respiratório e que possua indicação de encaminhamento ao alojamento conjunto ${ }^{8}$. Corroborando, evidencia-se que entre os pré-termos não submetidos à reanimação neonatal ou sem a necessidade de outras intervenções na sala de parto, apenas $14,3 \%$ realizaram o contato pele a pele logo após o nascimento ${ }^{22}$. 
No tocante ao AM precoce, o resultado de $34.7 \%$ dos RNPT se aproxima do percentual encontrado em um estudo com RN de maternidades da Rede Cegonha da região sudeste $31 \%$ e em outra pesquisa do estado do Rio de Janeiro com a mesma população $28 \%$. No entanto, em uma investigação com 22 RNPT de um hospital amigo da criança, constatouse que nenhum deles foi amamentado na primeira hora de vida ${ }^{22-24}$.

Apesar deste cuidado imediato se associar com menor risco de ocorrência de sepse, enterocolite necrosante e retinopatia da prematuridade, melhor desenvolvimento neuropsicomotor e redução das taxas de reinternações e tempo de hospitalização, é comum os RNPT apresentarem dificuldades para o AM precoce em função de condições clínicas do binômio, reflexo de sucção insatisfatório e imaturidade ao nascer ${ }^{25,26}$. Por outro lado, sabe-se que a implementação desta prática se depara com as divergências de condutas profissionais e a alta demanda assistencial aliada ao quantitativo insuficiente de recursos humanos ${ }^{27}$.

Dentre os fatores limitadores da implementação dos cuidados imediatos, o desconforto respiratório foi a complicação predominante nesta pesquisa, presente em 47,9\% dos RNPT. No entanto, este resultado difere dos achados de outros estudos, que revelaram um índice de $21,3 \%$ relacionado a esta intercorrência 28 , bem como evidenciaram a imaturidade pulmonar e a idade gestacional como os principais fatores ${ }^{13}$.

Sobre a hipotonia ao nascer, esta complicação se associou com dificuldades para o incentivo ao AM precoce, sendo constatada em 9,2\% do RNPT. Ressalta-se que este percentual é semelhante ao encontrado em uma investigação sobre aspectos clínicos de RNPT no nascimento, na qual 5,8\% da população com a mesma característica apresentou hipotonia ${ }^{29}$.

Diante da necessidade de estabilização do RNPT, houve um percentual expressivo de aspiração das vias áreas entre RNPT na sala de parto, corroborando com outros estudos ${ }^{20,30,31}$. Com base em evidências de alta qualidade, a OMS recomenda que, em RN que apresentem líquido amniótico claro e respiração espontânea após o nascimento, a aspiração nasofaríngea e orofaríngea não deve ser realizada ${ }^{32}$. Na prática, verifica-se que procedimentos sem respaldo científico de seus benefícios aos RNPT ainda têm adesão significativa entre profissionais da assistência perinatal ${ }^{20}$.

Neste contexto, observou-se que o oxigênio inalatório na sala de parto foi utilizado em $25,7 \%$ dos RNPT deste estudo, corroborando com dados de outra pesquisa, na qual $21,7 \%$ necessitaram da mesma terapia ${ }^{33}$. Ressalta-se que este procedimento é uma medida de suporte para ajudar na transição suave do sistema de troca de gases da placenta para o pulmão, tendo em vista a propensão dos RNPT às dificuldades respiratórias imediatamente após o nascimento ${ }^{9,13}$.

Ademais, constatou-se que a IOT e o clampeamento precoce foram os cuidados imediatos mais frequentes em RNPT muito pré-termos, com $66,7 \%(n=10)$ e $22,7 \%(n=17)$, respectivamente. Esta correlação também foi encontrada em outros estudos ${ }^{7,12,19}$, que também mostram a IOT como uma limitação à implementação de outros cuidados imediatos recomendados frente à instabilidade e a necessidade de transferência do RNPT para UTIN ${ }^{8}$.

Quanto ao desfecho, percebe-se que 67,9\% dos RNPT foram encaminhados ao AC, demonstrando uma boa estabilidade clínica na maioria dos casos de prematuridade moderada a tardia. Em RNPT com este perfil, observa-se que cuidados intensivos só são requeridos diante de intercorrências no parto ${ }^{16}$.

No tocante ao tempo de internação, este estudo encontrou a média de 15 dias, tempo superior ao evidenciado em uma pesquisa com RNPT moderados e tardios, onde a permanência de hospitalização prevalente foi de 1-3 dias $(39,7 \%)^{16}$. Ainda, considerando que $93,7 \%$ da população estudada recebeu alta hospitalar, nota-se que o percentual de mortalidade na instituição é baixo $(9,3 \%)$, resultado este similar aos $7,8 \%$ de prevalência encontrados em um estudo correlato $^{28}$.

Os achados desta pesquisa são importantes para a área da enfermagem e da atenção à saúde neonatal, uma vez que estudos de mapeamento dos cuidados imediatos ao RN na sala de parto viabilizam a identificação de fragilidades na assistência neonatal e oferecem subsídios para o planejamento de ações de educação permanente e a implementação de boas práticas, especialmente em relação aos RNPT.

\section{Limitações do estudo}

Como limitação do estudo, considera-se a qualidade dos registros e o delineamento retrospectivo, devido à restrição da documentação registrada previamente, o que impossibilitou uma observação do objeto estudado em tempo real.

\section{CONCLUSÃO}

Quanto às variáveis relacionadas ao RNPT, não houve predominância em relação ao sexo, a maioria apresentou peso e estatura compatíveis com AIG, boa vitalidade ao nascer no primeiro e quinto minuto, bem como foram classificados majoritariamente em pré-termos tardios quanto à idade gestacional. 
Evidenciou-se nos cuidados imediatos a prática do clampeamento do cordão umbilical precoce, aspiração de vias aéreas ao nascer com frequência e a necessidade da instalação de 02 inalatório para estabilização clínica. Nos RNPT submetidos à IOT, houve maior ocorrência maior entre prematuros muito pré-termos e tempo de clampeamento do cordão umbilical menor do que um minuto. A intercorrência que obteve destaque foi o desconforto respiratório, a maior parte foi encaminhado para internação em alojamento conjunto e teve como desfecho a alta hospitalar.

Assim, recomenda-se o planejamento e a implementação de ações de educação permanente para os profissionais que atuam na sala de parto, impulsionando práticas humanizadas e baseadas em evidências científicas, sobretudo por se tratar de um hospital de ensino.

\section{REFERÊNCIAS}

1. Kologeski TK, Strapasson MR, Schneider V, Renosto JM. Skin to skin contact of the newborn with its mother in the perspective of the multiprofessional team. Rev. enferm. UFPE on line [Internet]. 2017 [cited 2019 July 01]; 11(1):94-101. Available from: https://periodicos.ufpe.br/revistas/revistaenfermagem/article/download/11882/14340.

2. Croop, SEW, Thoyre SM, Aliaga, $S$ et al. The Golden Hour: a quality improvement initiative for extremely premature infants in the neonatal intensive care unit. J. Perinatol. [Internet]. 2020 [cited 2021 Sep 23]; 40(3):530-39. DOI: https://doi.org/10.1038/s41372-019-0545-0.

3. United Nations (US). The Millennium Development Goals report 2015. New York: United Nations [Internet]; 2015 [cited 2019 July 01]. Available from: https://www.un.org/millenniumgoals/2015_MDG_Report/pdf/MDG\%202015\%20rev\%20(July\%201).pdf.

4. Harriman TL, Carter B, Dail RB, Stowell KE, Zukowsky K. Golden Hour Protocol for Preterm Infants: A Quality Improvement Project. Adv. Neonatal Care [Internet]. 2018 [cited 2021 Jan 11]; 18(6):462-70. DOI: https://doi.org/10.1097/ANC.0000000000000554.

5. Departamento Científico de Neonatologia. Prevenção da prematuridade - uma intervenção da gestão e da assistência. Rio de Janeiro (RJ): Sociedade Brasileira de Pediatria [Internet]. 2017 [cited 2021 Jan 11]. Available from: https://www.sbp.com.br/fileadmin/user_upload/20399b-DocCient_-_Prevencao_da_prematuridade.pdf.

6. Ministério da Saúde (BR). Uma análise da situação de saúde e das doenças e agravos crônicos: desafios e perspectivas. Brasília: Ministério da Saúde [Internet]. 2018 [cited 2021 Jan 11]. Available from:

https://bvsms.saude.gov.br/bvs/publicacoes/saude_brasil_2018_analise_situacao_saude_doencas_agravos_cronicos_desafios perspectivas.pdf.

7. Agarwal P, Sharma A, Farooqi A, Natarajan G. Impact of different cord clamping strategies on short term neuromonitoring among preterm infants: a randomized, controlled trial. J. Perinatol. [Internet]. 2020 [cited 2021 Jan 10]; 40(7):1115-18. DOI: https://doi.org/10.1038/s41372-020-0684-3.

8. Sharma D. Golden hour of neonatal life: need of the hour. Matern Health Neonatol Perinatol [Internet]. 2017 [cited $2021 \mathrm{Mar}$ 20]; 3(16). DOI: https://doi.org/10.1186/s40748-017-0057-x.

9. Programa de Reanimação Neonatal. Reanimação do Prematuro <34 semanas em sala de parto: Diretrizes da Sociedade Brasileira de Pediatria - Versão 2016 com atualizações em maio de 2021. Rio de Janeiro (RJ): Sociedade Brasileira de Pediatria [Internet]. 2017 [cited 2021 Jan 11]. Available from: https://www.sbp.com.br/fileadmin/user_upload/DiretrizesSBP. ReanimacaoRN_Maior34semanas-MAIO_2021.pdf.

10. Silva JLP, Linhares FMP, Barros AA, Souza AG, Alves DS, Andrade PON. Factors associated with breastfeeding in the first hour of life in a baby-friendly hospital. Texto \& contexto enferm [Internet]. 2018 [cited 2019 July 01]; 27(4):e4190017. DOI: https://doi.org/10.1590/0104-07072018004190017.

11. .Antunes Ramos WM, Costa Aguiar BG, Conrad D, Pinto CB, Mussumeci PA. Contribution of obstetric nurse in good practices of childbirth and birth assistance. R. pesq. cuid. fundam. online [Internet]. 2018 [cited 2021 Mar 20]; 10(1):173-9. DOI: http://dx.doi.org/10.9789/2175-5361.2018.v10i1.173-179.

12. Nosherwan A, Cheung PY, Schmölzer GM. Management of Extremely Low Birth Weight Infants in Delivery Room. Clin. Perinatol. [Internet]. 2017 [cited 2021 Mar 20]; 44(2):361-75. DOI: https://doi.org/10.1016/j.clp.2017.01.004.

13. Nyqvist KH, Rosenblad A, Volgsten H, Funkquist EL, Mattsson E. Early skin-to-skin contact between healthy late preterm infants and their parents: an observational cohort study. Peer J [Internet]. 2017 [cited 2021 Mar 20]; 30(5):e3949. DOI: https://doi.org/10.7717/peerj.3949.

14. Williams JE, Pugh Y. The Late Preterm: A Population at Risk. Crit. Care Nurs. Clin. North Am. [Internet]. 2018 [cited $2021 \mathrm{Mar}$ 20]; 30(4):431-43. DOI: https://doi.org/10.1016/j.cnc.2018.07.001.

15. Benicio AL et al. Care to the child less than one year old: nursing practice perspective about child care. Rev. enferm. UFPE on line [Internet]. 2016 [cited 2021 Jan 11]; 10(2):576-84. Available from: https://periodicos.ufpe.br/revistas/revistaenfermagem/article/viewFile/10992/12344.

16. Descovi MH, Jantsch LB, Rosa N, Kegler JJ, Neves ET. Resuscitation of moderate and late preterm babies in the delivery room: associated factors. Acta Paul. Enferm. [Internet]. 2020 [cited Feb 22]; 33:1-8. DOI: https://doi.org/10.37689/actaape/2020ao0134.

17. Rabe H, Gyte GM, Diaz-Rossello JL, Duley L. Effect of timing of umbilical cord clamping and other strategies to influence placental transfusion at preterm birth on maternal and infant outcomes. Cochrane Database Syst. Rev. [Internet]. 2019 [cited 2021 Jan 10]; 9(9):CD003248. DOI: https://doi.org/10.1002/14651858.CD003248.pub4. 
18. Katheria A, Reister F, Essers J, et al. Association of umbilical cord milking vs delayed umbilical cord clamping with death or severe intraventricular hemorrhage among preterm infants. JAMA [Internet]. 2019 [cited 2021 Jan 11]; 322(19):1877-86. DOI: https://doi.org/10.1001/jama.2019.16004.

19. Duley L, Dorling J, Pushpa-Rajah A, et al. Randomised trial of cord clamping and initial stabilisation at very preterm birth. Arch. Dis. Child Fetal Neonatal Ed. [Internet]. 2018 [cited 2021 Feb 11]; 103(1):F6-F14. DOI: https://doi.org/10.1136/archdischild2016-312567.

20. Barros GM, Dias MAB, Gomes Junior SCS. The use of good care practices to newborns in the fi rst hour of life in different childbirth care models. Rev. Soc. Bras. Enferm. Ped. [Internet]. 2018 [cited 2021 Jan 11]; 18(1):21-8. DOI: http://dx.doi.org/10.31508/1676-3793201800004.

21. Campos PM, Gouveia HG, Strada JKR, Moraes BA. Skin-to-skin contact and breastfeeding of newborns in a university hospital. Rev. Gaúcha Enferm. [Internet]. 2020 [cited 2021 Mar 23]; 41(spe):e20190154. DOI: https://doi.org/10.1590/19831447.2020.20190154.

22. Lima LS, Reis EAF, Silva EM, Moura JPG. Nursing care in the thermo-regulation of preterm newborns: an integrative review. Cogitare enferm. [Internet]. 2020 [cited 2021 Sep 24]; 25. DOI: http://dx.doi.org/10.5380/ce.v25i0.70889.

23. Balaminut T, Sousa MI, Gomes ALM, Christoffel MM, Leite AM, Scochi CGS. Breastfeeding in premature infants discharged from baby-friendly hospitals in southeastern Brazil. Rev. Eletr. Enf. [Internet]. 2018 [cited 2021 Mar 21]; 20(20). DOI: https://doi.org/10.5216/ree.v20.50963.

24. Gomes MASM, Esteves- Pereira AP, Bittencourt SDA, Augusto LCR, Lamy-Filho F, Lamy Z et al. Care for healthy newborns in Brazil: are we making progress in achieving best practices?. Ciênc. Saúde Colet. [Internet]. 2021 [cited 2021 Mar 26]; 26(3): 85974. DOI: https://doi.org/10.1590/1413-81232021263.26032020.

25. Benatti Antunes M, Demitto MO, Gramazio Soares L, Trindade Radovanovic CA, Harumi Higarashi I, Ichisato SMT et al. Breastfeeding within the first hour after birth: knowledge and practice of multidisciplinary team. Av. Enferm. [Internet]. 2017 [cited 2021 Mar 20]; 35(1):19-29. DOI: https://doi.org/10.15446/av.enferm.v35n1.43682.

26. Quigley M, McGuire W. Formula versus donor breast milk for feeding preterm or low birth weight infants. Cochrane Database Syst Rev. [Internet]. 2014 [cited 2021 Jan 10]; (4):CD002971. DOI: https://doi.org/10.1002/14651858.CD002971.pub3.

27. Smith ER, Hurt L, Chowdhury R, et al. Delayed breastfeeding initiation and infant survival: A systematic review and metaanalysis. PLoS One. [Internet]. 2017 [cited 2021 Feb 11]; 12(7):e0180722. DOI: https://doi.org/10.1371/journal.pone.0180722.

28. Benatti Antunes M, Demitto MO, Gramazio Soares L, Trindade Radovanovic CA, Harumi Higarashi I, Ichisato SMT et al. Breastfeeding within the first hour after birth: knowledge and practice of multidisciplinary team. Av. Enferm. [Internet]. 2017 [cited 2021 Mar 20]; 35(1):19-29. DOI: http://dx.doi.org/10.15446/av.enferm.v35n1.43682.

29. Almeida B, Couto RHM, Trapani Júnior A. Prevalence and factors associated with death in interned prematures. Arq. Catarin Med. [Internet]. 2019 [cited 2021 Oct 11]; 48(4):35-50. Available from:

http://www.acm.org.br/acm/seer/index.php/arquivos/article/view/512.

30. Araújo e Lima RS, Alves TM, Bezerra BRS, Dias MB et al. Relation between palmar and plantar gripping reflections and clinical aspects at the birth of premature babies. Braz. J. of Develop. [Internet]. 2020 [cited 2021 Oct 11]; 6(7):49533-44. DOI: https://doi.org/10.34117/bjdv6n7-558.

31. Ledo BC, Góes FGB, Santos AST, Pereira-Ávila FMV, Silva ACSS, Bastos MPC. Factors associated with care practices for newborns in the delivery room. Esc. Anna Nery Rev. Enferm. [Internet]. 2021 [cited 2021 Mar 24]; 25(1):e20200102. DOI: https://doi.org/10.1590/2177-9465-ean-2020-0102.

32. Abdala LG; Cunha MLC. Skin-to-skin contact between mother and newborn and breastfeeding in the first hour of life. Clin. biomed. res. [Internet]. 2019 [cited 2021 Jan 10]; 38(4):356-60. DOI: https://doi.org/10.4322/2357-9730.82178.

33. Word Health Organization. WHO recommendations: intrapartum care for a positive childbirth experience. Geneva: WHO [Internet]. 2018 [cited 2021 Oct 11]. Available from: http://apps.who.int/iris/bitstream/handle/10665/260178/9789241550215-eng.pdf?sequence=1.

34. Lourenço N, Fernandes M, Gomes C, Resende C. Neonatal morbidity of late preterm compared with early term neonates. Sci Med. [Internet]. 2017 [cited 2021 Oct 11]; 27(1):25876 DOI: http://doi.org/10.15448/1980-6108.2017.1.25876. 